\title{
La anotación como estrategia para mejorar la expresión escrita en inglés
}

Asunción Jaime $^{\mathrm{a}}$, Cristina Pérez Guillot ${ }^{\mathrm{b}}$ y Felix Buendia ${ }^{\mathrm{c}}$

${ }^{a}$ Dto Lingüística Aplicada - UPV, ajaime@upvnet.upv.es; ${ }^{b}$ Dto Lingüística Aplicada - UPV mcperez@upvnet.upv.es; y ${ }^{c}$ Dpto. de Informática de Sistemas y Computadores - UPV fbuendia@disca.upv.es

\begin{abstract}
The introduction of new technology has dramatically changed the paradigm of communication, particularly among young people who are currently our university students.

This new form of communication, such as SMS, WhatsApp, Twitter, e-mail, etc., is characterized by the use of short messages and lack of a defined organizational text structure. As a result, more and more candidates fail the writing section in English language proficiency exams.

It is thus clear that there is a need to explore new educational approaches that favor the development of more efficient writing strategies than traditional methods.

Text annotation is a technique that involves adding a note or gloss to a text, which may include highlights or underlining, comments, etc. Among the advantages of this technique we can mention that it favors collaborative learning, increases student motivation to work together, and allows developing a comprehensive picture of text structure and its linguistic components.

In this paper we present a practical application of the technique of text annotation using Hypothes.is software that has been carried out at the School of Business Administration with 4th year students taking the subject English for Business Communication
\end{abstract}

Keywords: text annotation , writing, English, teaching-learning , collaborative learning

\footnotetext{
Resumen

La introducción de las nuevas tecnologías ha cambiado de forma radical el paradigma de la comunicación, particularmente entre los jóvenes que son actualmente nuestros alumnos universitarios.

Esta nueva forma de comunicación, como SMS, WHATSAPP, TWITTER, email, etc, se caracteriza por la brevedad del mensaje y carencia de una estructura organizativa definida. Como consecuencia, cada vez hay más candidatos que no superan la prueba de expresión escrita en exámenes de competencia lingüistica de inglés.
} 
Queda asi patente que es necesario explorar nuevos enfoques educativos que favorezcan el desarrollo de estrategias de escritura en inglés más eficientes que los métodos tradicionales.

La anotación es una técnica que consiste en ir añadiendo notas a un texto, tales como comentarios, sugerencias, observaciones, etc. Entre las ventajas de esta técnica podemos destacar la de favorecer el aprendizaje colaborativo, incrementado la motivación de los estudiantes al trabajar en equipo, lo que permite desarrollar una visión más completa de la estructura textual y profundizar en sus componentes lingüisticos.

'En este trabajo presentamos una aplicación práctica de la técnica de anotación mediante la aplicación Hypothes.is que se ha llevado a cabo en la Facultad de ADE con alumnos de $4^{\circ}$ de grado que cursan la asignatura Inglés para la Comunicación Empresarial

Palabras clave: anotación textual, expresión escrita, inglés, enseñanzaaprendizaje, aprendizaje colaborativo

\section{Introducción}

La introducción de las nuevas tecnologías ha cambiado de forma radical el paradigma de la comunicación, particularmente entre los jóvenes que desde su infancia han convivido con los dispositivos electrónicos y que actualmente son nuestros alumnos universitarios. Esta nueva forma de comunicación, como SMS, WSAP, TWITTER, e-mail, etc, se caracteriza por la brevedad del mensaje y carencia de una estructura organizativa definida. Como consecuencia, los jóvenes tienen problemas a la hora de redactar textos que comporten cierta longitud y complejidad, especialmente si lo tenen que hacer en un idioma extranjero, como lo demuestra el procentaje de candidatos que no superan la prueba de expresión escrita en exámenes de competencia lingüística de inglés y cuyo porcentaje aumenta en los niveles más altos del MCER al incrementarse la complejidad de las tareas de escritura ${ }^{1}$

Queda así patente que es necesario explorar nuevos enfoques educativos que favorezcan el desarrollo de estrategias de escritura en inglés más eficientes y eficaces que los métodos tradicionales.

La anotación es una técnica que consiste en ir añadiendo notas a un texto, tales como comentarios, sugerencias, indicaciones, observaciones, etc. 'Text Annotation is the practice and the result of adding a note or gloss to a text, which may include highlights or underlining, comments, footnotes, tags, and links' (Wikipedia, 2016). Esta técnica se aproxima mejor a la forma de comunicarse actual al consistir en la elaboración de frases o comentarios breves con un objetivo concreto, dependiendo de la tarea que se esté llevando a cabo.

\footnotetext{
${ }^{1}$ En las pruebas de certificación de competencia lingüística de inglés CertAcles-UPV B2 de diciembre de 2015, la prueba de expresión escrita fue la presentó un mayor porcentaje de suspensos $(28,26 \%)$, sequida por la de comprensión auditiva $(25,54 \%)$, expresión oral $(13,59 \%)$ y comrensión escrita $(8,15)$
} 
Diversos autores han señalado los beneficios de esta técnica para mejorar las habilidades de la compresión y expresión escrita (Yayli, 2012; Brown, 2007; Stuart, 2014; PorterO'Donnell, 2004; Wolfe, 2000; Wolfe, 2002 ). Entre las ventajas de la anotación textual podemos destacar la de favorecer el aprendizaje colaborativo, incrementado la motivación de los estudiantes al trabajar en equipo, lo que permite desarrollar una visión más completa de la estructura textual y profundizar en sus componentes gramaticales y retóricos: 'One possible way of building language awareness in writing is annotation writing through which students achieve enhanced consciousness of the forms and the functions of the language and the awareness of the limitations of the type of writing they are engaged with'(Yayli, 2012:45).

Existen diversos tipos de herramientas que aprovechan la técnica de anotaciones como parte de un proceso de análisis de textos escritos. Entre estas herramientas encontramos Dexter (Garretson, 2006) o SACODEYL (Pérez-Paredes \& Alcaraz-Calero, 2009) para la anotación en corpus lingüísticos. En ambos casos, la técnica de anotación se enfoca al análisis lingüístico con objetivos ya sea pedagógicos o de investigación. En este trabajo, se trata de profundizar en métodos colaborativos donde las herramientas de anotación online permitan fomentar el intercambio de conocimiento. Para ello, se han examinado varias herramientas como Annotation Studio ${ }^{2}$ o Hypothes.is ${ }^{3}$ basadas en una librería JavaScript que ofrece múltiples funcionalidades de anotación. Finalmente, se ha seleccionado Hypothes.is como entorno para realizar diversas pruebas que permitan mostrar las posibilidades de utilizar anotaciones y su impacto a la hora de mejorar la expresión escrita. Una de sus principales ventajas es la de poder anotar diversos tipos de documentos online (pdf o html por ejemplo) sin necesidad de disponer de un espacio de almacenamiento propio para dichos documentos.

Así mismo, la utilización del entorno de anotación Hypothes.is disponible on-line le permite al estudiante acceder a ella en cualquier momento y lugar, pudiendo de este modo establecer una planificación personalizada de las tareas de aprendizaje y realizar las anotaciones y comentarios de acuerdo con su sistema de aprendizaje.

De este modo, a diferencia de otras estrategias tradicionales más pasivas, el proceso de anotar un texto y compartir los comentarios con otros miembros de la clase lo convierte en un proceso activo y participativo que ayuda a mejorar las estrategias de escritura y favorecen el desarrollo del aprendizaje personal (Dörnyei, 2007).

En este trabajo presentamos una aplicación práctica de la técnica de anotación que se ha llevado a cabo en la Facultad de ADE con alumnos de $4^{\circ}$ de grado que cursan la asignatura Inglés para la Comunicación Empresarial (ICE) nivel B2 utilizando la herramienta on-line de anotación Hypothes.is

\footnotetext{
${ }^{2}$ http://www.annotationstudio.org/

3 https://hypothes.is/ is a 501(c) open-source software project that aims to collect comments about statements made in any webaccessible content
}

(c)) EY-NC-ND 2016, Universitat Politècnica de València

Congreso In-Red (2016) 


\section{Objetivos}

El objetivo principal es desarrollar técnicas de escritura en inglés que sean eficientes y se adapten a las nuevas formas de comunicación mediante el uso de la anotación textual colaborativa.

Como objetivos de aprendizaje, podemos destacar el trasvasar algunas de las estrategias de comunicación de la vida cotidiana al entorno académico. Así, al convertirse el proceso de aprendizaje en un proceso activo compartido, se incrementa la implicación del estudiante en la tarea, con lo que presta mas atención a las estructuras lingüísticas y gramaticales y potencia la memorización y desarrollo de estrategias textuales:

De este modo se incrementa el interés y la motivación del estudiante para la realización de las tareas lingüísticas y reflexiona sobre la importancia del trabajo en equipo ya que las anotaciones de los compañeros ayudarán a cada estudiante a complementar su conocimiento inicial de aspectos gramaticales, léxicos, retóricos y textuales.

En cuanto a los objetivos didácticos concretos de la tarea que presentamos, al tratarse de la escritura de una carta de solicitud de empleo, podemos mencionar los siguientes:

- Conocer la estructura general que se debe seguir al redactar una carta solicitando empleo

- Aprender las fórmulas estándar que se utilizan en inglés para cada sección de una carta de este tipo

- Identificar elementos léxicos, gramaticales y retóricos que se suelen emplear en la redacción de cartas de solicitud de empleo en inglés

- Desarrollar patrones mentales de los distintos tipos textos a los que se deberán enfrentar los alumnos cuando se incorporen al mundo laboral

Así pues, mediante la técnica de la anotación se pueden lograr estos objetivos puesto que, como ya indicaba Beck (1982: 322) 'develops authorial maturity, enhancing effectiveness of teaching and helping student-teacher communication'

\section{Desarrollo de la tarea}

Debido a los problemas detectados en los estudiantes a la hora de redactar textos en inglés, como lo demuestran los resultados en exámenes oficiales de certificación de competencia lingüística, en el presente trabajo presentamos la técnica de la anotación para mejorar las estrategias de escritura en inglés de los estudiantes de la Facultad de Administracion de Empresas de la UPV que están cursando la asignatura de ICE correspondiente a $4^{\circ}$ curso del grado de Administración y Gestión de Empresas de la Facultad de Administración de Empresas (FADE), y que tiene una carga docente de 4,5 créditos.

Esta asignatura es de carácter obligatorio, siendo la continuación de la asignatura ofrecida de forma optativa durante el curso anterior Inglés para la Gestión Empresarial (IGE) nivel B1. La mayoría de los alumnos han cursado la asignatura optativa con lo que están ya familiarizados con el vocabulario y la estructuras del lenguaje del mundo de los negocios, 
pero sin duda necesitan consolidar sus conociemientos para poder expresarse por escrito con un registro adecuado a un nivel B2 consolidado.

Dentro de la tipología textual, tanto en exámenes oficiales de competencia lingüística como en su vida profesional, los estudiantes de Administración de Empresas deberán redactar cartas con distintos objetivos, por lo que conocer los modelos que caracterizan cada una de ellas será de utilidad al alumno no solo durante su vida académica sino también en su posterior vida laboral.

En este trabajo nos centraremos en la redacción de cartas de solicitud de empleo ya que son particularmente relevantes pues es una tarea que los alumnos sin duda deberán realizar al finalizar sus estudios, luego la motivación es mucho mayor.

A continuación describimos cómo hamos utilizado la técnica de la anotación para ayudar a los estudiante a redactar cartas de solicitud d eempleo

- Tras una breve introducción de la tipología de cartas más frecuentes en el ámbito de organización empresarial, se les explica a los alumnos el uso de la herramienta Hypothes.is y se activa el grupo en el programa para que los alumnos puedan ir subiendo sus comentarios, como se muestra en la figura 1:

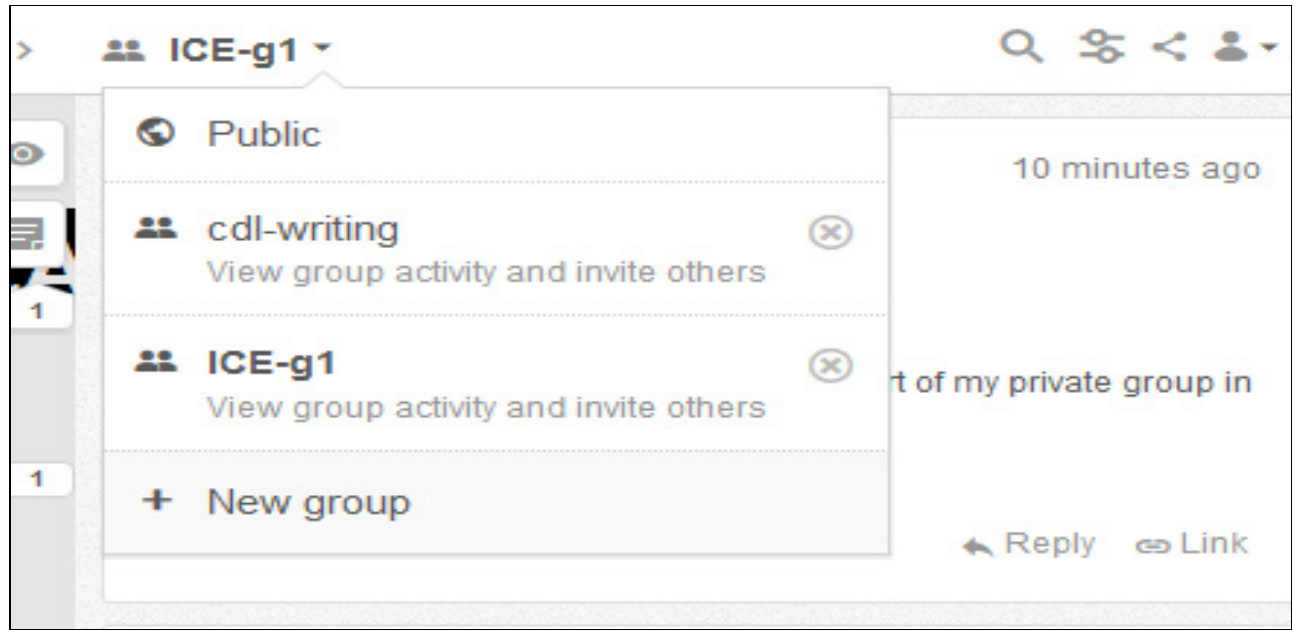

Fig. 1 Activación del grupo con el programa Hypothes.is

- Se sube al programa dos modelos de carta y se les pide a los alumnos que anoten los distintos apartados que conforman una carta de solicitud de empleo. Asímismo, los alumnos deben identificar el uso de determinadas fórmulas o expresiones lingüísticas y léxico específico para ese tipo concreto de carta. Y por último los alumnos también deben anotar expresiones alternativas, reflexionar y hacer comentarios sobre las estructura de la carta, los tiempos gramaticales, uso de voz activa o pasiva $u$ otros elementos sintácticos o gramaticales que les hayan llamado la atención. 
En el ejemplo (figura 2), presentamos los dos modelos de carta sobre los que deben trabajar los alumnos e ir anotando sus comentarios y sugerencias usando el entorno Hypothes.is:

\section{SAMPLE A}

Dear Mr Brown,

Please find enclosed my CV in application for the post advertised in the Guardian on 30 November.

As you can see, I have had extensive vacation work experience in office environments, the retail sector and service industries, giving me varied skills and the ability to work with many different types of people. I believe I could fit easily into your team.

I am a conscientious person who works hard and pays attention to detail. I'm flexible, quick to pick up new skills and eager to learn from others. I also have lots of ideas and enthusiasm. I'm keen to work for a company with a great reputation and high profile like yours.

Thank you for taking the time to consider this application and I look forward to hearing from you in the near future.

Yours sincerely

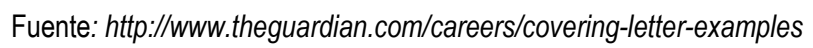

\section{SAMPLE B}

Subject : Personal Assistant to Overseas Sales Manager

I'm writing to express my interest in the Personal Assistant to Overseas Sales Manager position listed on your advertisement. I have been working for two years as a receptionist and a secretary so I have a strong experience in secretarial duties.

Then, as I completed all my English courses at college, my knowledge of this language fits perfectly for this job position. I can also speak fluently Italian because I was living in Roma during the summer of 2008.

Moreover, I would like to add that l've been working for a travel agency during these last six months. That's why the opportunity to travel abroad offered by this position sounds really interesting to me, as I am used to deal with travelling issues.

Thank you for your consideration

Fig.2. Modelos de carta de solicitud de empleo usadas en la tarea

Y las siguientes imágenes (figura 3) ilustran cómo los alumnos van anotando sus comentarios, sugerencias y observaciones en la plataforma Hypothes.is:

(c) EY-NC-ND 2016, Universitat Politècnica de València

Congreso IN-RED (2016) 


\section{SAMPLE $A$}

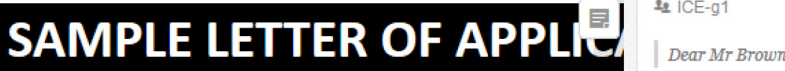

Subject: Cover letter example

\section{Dear Mr Brown,}

[Introduction] I am writing to enquire if you have any vacancies in your company. I enclose my CV for your information.

[Working experience] As you can see, I have had extensive vacation work experience in office environments, the retail sector and service industries, giving me varied skills and the ability to work with many different types of people. I believe I could fit easily into your team.

[Personal skills] I am a conscientious person who works hard and pays attention to detail. I'm flexible, quick to pick up new skills and eager to learn from others. I also have lots of ideas and enthusiasm. I' $m$ keen to work for a company with a great reputation and high profile like [insert company name

[Closing] I have excellent references and would be delighted to discuss any possible vacancy with you at your convenience. In case you do not have ky

About salutation, could you mention, by replying this annotation, some formula examples when letter recipients are unknown?

2 replies Edit Delete $\pitchfork$ Reply $\Leftrightarrow$ Link

- curso32 13 minutes ag

is $\mid \mathrm{CE}-\mathrm{g}$

We can begin with "Dear Sir,"/"Dear Madam," but, if we do not know the sex of the person we are writing to.

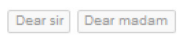

1 reply

curso31

$\hookrightarrow$ Reply $\Leftrightarrow$ Link

ts ICE-g1

We can simply write "Dear Sir or Madam". Any other suggestion?

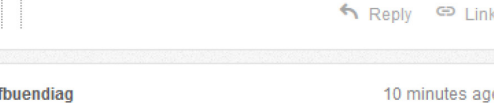

Yours sincerely

\section{SAMPLE B}

(1) Public .

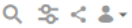

curso31

54 minutes ago

\section{SAMPLE LETTER OF APPLIE!}

Subject: Personal Assistant to Overseas Sales Manager

I'm writing to express my interest in the Personal Assistant to Overseas Sales Manager position listed on your advertisement. I have been working for two years as a receptionist and a secretary so I have a strong
experience in secretarial duties.

Then, as I completed all my English courses at college, my knowledge of this language fits perfectly for this job position. I can also speak fluently Italian because I was living in Roma during the summer of 2008.

Moreover, I would like to add that I've been working for a travel agency during these last six months. That's why the opportunity to travel abroad offered by this position sounds really interesting to me, as I am used to deal with travelling issues.

Thank you for your consideration

Fig. 3 Ejemplo de uso del programa Hypothes.con anotaciones de los alumnos

- En la siguiente fase, se hace una puesta en común de las anotaciones y conclusiones a las que ha llegado cada grupo y el profesor resume y aclara dudas (figura 4).

\section{SAMPLE A}

Dear Mr Brown, (formula encabezamiento)

\section{(cc) BY-NC-ND 2016, Universitat Politècnica de València}




\begin{abstract}
PARRAFO 1: INTRODUCCION: Please (formula introducción) find enclosed my CV formula introducción in application for the post advertised in formula introducción the Guardian on 30 November.

PARRAFO 2: EXPERIENCIA PERSONAL RELACIONADA CON EL PUESTO: As you can see, I have had(tiempo verbal: present perfect) extensive (adj) vacation work experience (voc) in (prep)office environments, the retail sector and service industries, giving me varied skills (useful expression) and the ability to work useful expression with many different types of people. I believe (formula ) I could fit (formula) easily into (prep )your team (voc).
\end{abstract}

PARRAFO 3: HABILIDADES PERSONALES I am a(formula) conscientious person who (relative clause) works hard (expression) and pays attention to detail. I'm flexible, quick to pick up new skills and eager to learn from others (useful expressions). I also (connector)have lots of ideas and enthusiasm (adj) I'm keen to work(useful expression) for (prep)a company with(prep) a great reputation (useful expression)and high profile like yours. (useful expression)

PARRAFO 4: DESPEDIDA Thank you for taking the time to consider this application (formula despedida) and I look forward to hearing from you in the near future (formula despedida).

Yours sincerely (formula despedia)

\title{
SAMPLE B
}

Subject : Personal Assistant to Overseas Sales Manager

PARRAFO 1: INTRODUCCION:I'm writing to express my interest in formula introducción the Personal Assistant to Overseas Sales Manager position (voc) listed on your advertisement (VOC). PARRAFO 2: EXPERIENCIA PERSONAL RELACIONADA CON EL PUESTO I have been working(tiempo verbal: present perfect for (prep) two years as a (connector) receptionist and a secretary so (connector) I have a strong (adj) experience (voc) in (prep)secretarial duties.

PARRAFO 3: HABILIDADES PERSONALES Then, as (connector) I completed (verb tense: simple past) all my English courses at (prep) college, my knowledge of this language (expression) fits perfectly (voc) for (prep) this job position (voc). I can also speak fluently Italian because I was living in Roma during the summer of 2008.

Moreover (connector), I would like to (formula) add that (voc) I've been working for a travel agency during these last six months. That's why the opportunity to (voc) travel abroad offered by this position (expression) sounds really interesting to me (formula), as (connector) I am used to (expression) deal with travelling issues.

PARRAFO 4: DESPEDIDA Thank you for your consideration (formula)

Fig. 4 Resumen de las observaciones realizadas por los alumnos

- A continuación cada grupo escribe una carta de solicitud de empleo y se la pasa a otro de los grupos, que realizan anotaciones tanto a nivel de comentario como lingüísticas. Un ejemplo de tarea es la siguiente (figura 5):

(c) B EY-NC-ND 2016, Universitat Politècnica de València

Congreso IN-RED (2016) 
TASK

Read this advert for a job. You would like to apply for it and you have written some notes about your experience.

Then, using all the personal notes below, write a letter of application (120-140 words

Personal Assistant to Overseas Sales Manager

We are currently seeking someone to work in a busy and expanding department. You will have experience in secretarial duties. Good knowledge of English. Opportunities for promotion.

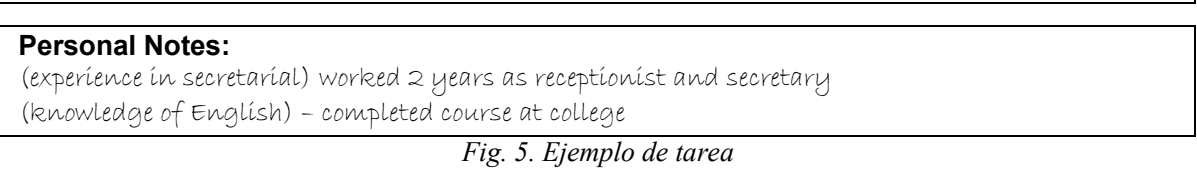

- Con las anotaciones proporcionadas, el grupo deber reescribir su carta para mejorar sus estrategias ya que como idican Zhang, y Litman, (2015: 133) 'Rewriting is considered as an important factor of successful writing'.

Este es un ejemplo con las anotaciones realizadas por otro grupo(figura 6):

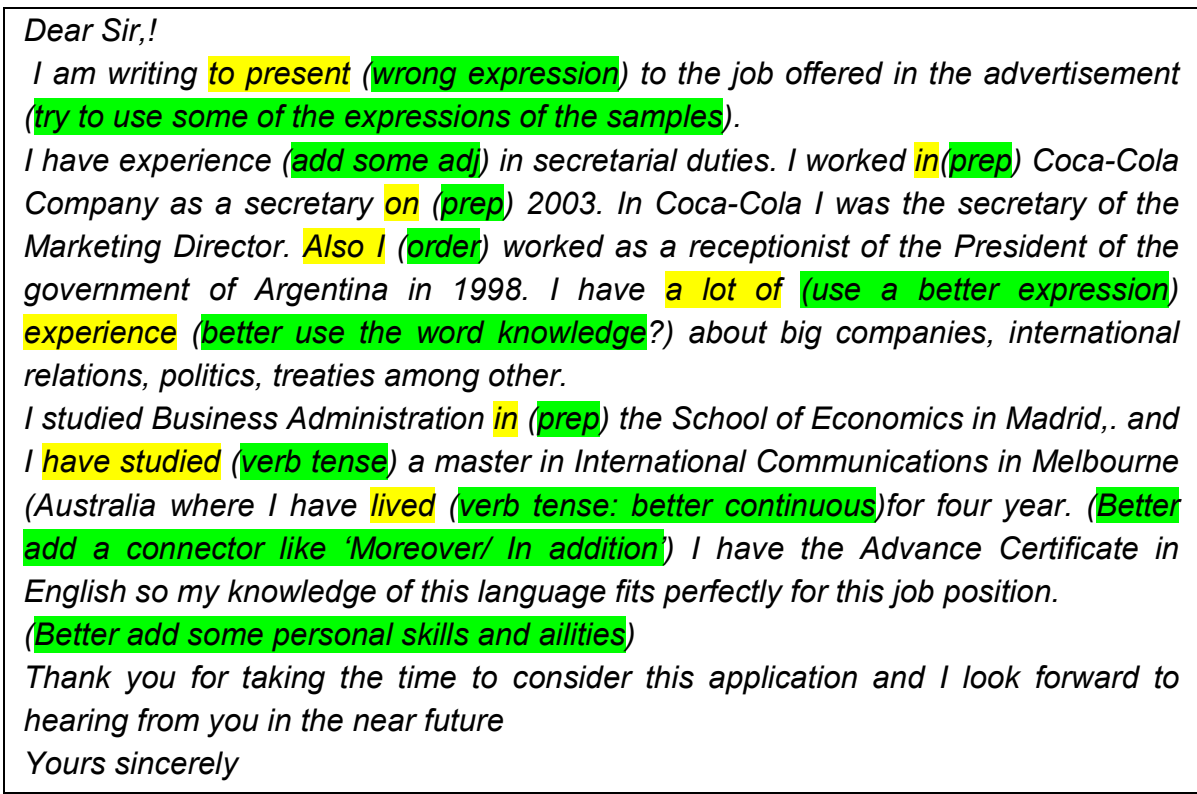

Fig. 6 Resumen de las anotaciones realizadas por los alumnos para mejorar el modelo de carta

El dinamismo y flexibilidad de la herramienta Hypothes.is ayudó a los alumnos a ir resolviendo sus dudas con los comentarios aportados por otros alumnos y a hacer una reflexión más profunda sobre la estructura y características lingüísticas de este tipo de cartas, mejorando sus habilidades de escritura.

\section{Resultados}

La realización de la tarea de escribir y reescribir una carta de solicitud de empleo utilizando las anotaciones, comentarios y sugerencias aportadas por otros compañeros reveló una

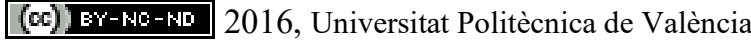

Congreso In-Red (2016) 
mayor concienciación e interés de los alumnos por los distintos aspectos lingüísticos y textuales que se emplean en la redacción de cartas en inglés.

De hecho, semanas después se les pidió que elaborasen una carta semejante, con excelentes resultados, lo que también demuestra que este tipo de estrategias colaborativas despiertan el interés y la motivación de los alumnos y les ayuda a reflexionar mas profundamente sobre aspectos idiomáticas que utilizando otros enfoques más tradicionales

Además, el hecho de que la técnica de la anotación se asemeje a su forma de comunicarse cotidiana asi como el uso de aplicaciones on-line que le permiten al alumno trabajar en entornos fuera de la clase les ayuda a utilizar estrategias que les son familiares para el aprendizaje del inglés.

No obstante, es cierto que la práctica de esta técnica supone un mayor tiempo de deciación por parte del alumno. Por ello solo hemos incorporado alguna tareas muy concretas basadas en la técnica de la anotación y con el grupo de flip-teaching, ya que puede gestionarse el tiempo de forma más abierta que en el caso de una clase convencional.

\section{Conclusiones}

La anotación textual es una ténica con gran potencial para el aprendizaje de un idioma extranjero como alternativa a otros sistemas docentes convencionales al utilizar en su proceso de aprendizaje estrategias de comunicación que son familiares a los estudiantes universitarios.

En el presente trabajo hemos utilizado esta técnica para fomentar el desarrollo de estrategias de escritura en inglés ya que es una de las destrezas comunicativas que presentan más dificultades a nuestros alumnos. En concreto hemos descrito cómo se puede incorporar la técnica de la notación en la realización de tareas de escritura de cartas comerciales en inglés.

Los resultados de la experiencia han sido positivo ya que los alumnos han adquirido de forma eficaz y eficiente determinadas estrategias de escritura de cartas al mismo tiempo que han aprendido elementos léxicos, sintácticos y gramaticales : language awareness relies on the learners paying conscious attention to instances of language in an attempt to discover and articulate patterns and language use '(Cheng, 2006:84).

Además, dado que para la práctica de esta técnica hemos utilizado la aplicación on-line Hypothes.is es un recurso didáctico muy flexible temporal y espacialmente, fomentando la autonomía del aprendizaje y la gestión personalizada del tiempo : 'the student's self monitoring increases autonomy in the learning of writing by giving learners' control over their own writing (Cresswell, 2000).

Por otra parte, fomentar tareas colaborativas y de reflexión que combinan aspectos didácticos y formativos le reportan beneficios al estudiante no solo durante su periodo académico sino posteriormente en su vida profesional.

Si bien en el presente trabao nos hemos centrado en la aplicación de la anotaión textual para el desarrollo de estrategias de escritura en inglés, es una técnica que se ha aplicado a otras

(c)) EY-NC-ND 2016, Universitat Politècnica de València 
disciplinas como en biomedicina (Kirsch et al. , 2006) o en iencias políticas (Cardie y Wilkerson, 2008), corroborando el potencial de esta técnica tanto para el aprendizaje como para la investigación.

Así pues, la anotación textual ha evidenciado ser una valiosa técnica complementaria de aprendizaje, en consonancia con los resultados obtenidos por otros autores, como Storch y Tapper (1997: 263) 'annotation writing can be seen as a reliable research tool to highlight different areas of student satisfaction and concerns about their own writing'

No obstante, como afirman algunos autores (Yayli, 2012; Zhang y Litman, 2015) es una técnica que ofrece buenos resultados pero que debe alternarse con el empleo de un amplio abanico de estrategias y métodos de aprendizaje

\section{Referencias}

BECK, J.P. (1982). “Asking students to annotate their own papers" en College Composition and Communication, 33(3), 322-326

Brown, M. (2007) "I'll Have Mine Annotated, Please: Helping Students Make Connections with Texts" en English Journal. 96 (4).

CARDIE, C. Y WILKERSON, J. (2008). "Text annotation for political science research", en Journal of Information Technology \& Politics, vol 5, 1-6

CHENG, A. (2006). "Understanding learners and learning in ESP genre-based writing instruction" en English for Specific Purposes, 25, 76-89.

CREsswell, A. (2000). "Self-monitoring in student writing: Developing learner responsibility" en ELT Journal, 54(3), 235-244.

DÖRNYEI, Z. (2007). Research methods in applied linguistics: Quantitative, qualitative and mixed methodologies. Oxford: Oxford University Press.

GARRETSON, G. (2006). Dexter: "Free tools for analyzing texts". En Pérez-Llantada AurÃa, M. C., R. Pló Alastrué \& C. P. Neumann, Academic and professional communication in the 21st century: genres, rhetoric and the construction of disciplinary knowledge. Proceedings of the 5th International AELFE Conference.

KIRSCH, H. ET AL. (2006) "Distributed modules for text annotation and IE applied to the biomedical domain", en International Journal of Biomedical Informaics, vol 75 (6), 496500

PÉREZ-PAREDES, P. y AlCARAZ CALERO.J.M. (2009). "Developing annotation solutions for online data-driven learning" en ReCALL 21 (1), 55-75.

PORTER-O'DONNELL, C. (2004) "Beyond the Yellow Highligher: Teaching Annotation Skills to Improve Reading Comprehension" en English Journal. 93 (5), 82-89.

StORCH, N., Y TAPPER, J. (1997). "Student annotations: What NNS and NS university students say about their own writing" en Journal of Second Language Writing, 6(3), 245264.

StuART, D. (2014). "Purposeful Annotation: A Close Reading Strategy that Makes Sense

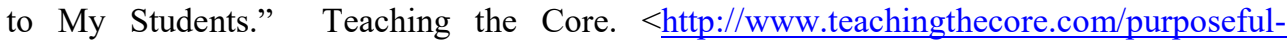
annotation-close-reading/> [Consulta 8 Marzo 2016]

(c) EY-NC-ND 2016, Universitat Politècnica de València

Congreso In-Red (2016) 
WIKIPEDIA. "Text annotation" $<$ https://en.wikipedia.org/wiki/Text_annotation $>$ [Consulta 6 Febrero 2016]

Wolfe, J. (2000) "Effects of Annotations on Student Readers and Writers" en Proceedings of the fifth ACM conference on Digital libraries, 19-26

WOLFE, J. (2002). “Annotation technologies: A software and research review" en Computers and Composition, $19: 471-497$

YAYLI, D. (2012). "Tracing the Benefits of Self Annotation in Genre-based Writing" en The Journal of Language Teaching and Learning, 2(1), 45-58

ZHANG, F. Y LITMAN, D. (2015). “Annotation and Classification of Argumentative Writing Revisions" en Proceedings of the Tenth Workshop on Innovative Use of NLP for Building Educational Applications, 133-143. 